\title{
Quasi-zero lattice mismatch and band alignment of BaTiO3 on epitaxial (110)Ge
}

M. K. Hudait, Y. Zhu, N. Jain, D. Maurya, Y. Zhou, and S Priya

Citation: Journal of Applied Physics 114, 024303 (2013); doi: 10.1063/1.4813226

View online: http://dx.doi.org/10.1063/1.4813226

View Table of Contents: http://scitation.aip.org/content/aip/journal/jap/114/2?ver=pdfcov

Published by the AIP Publishing

\section{Articles you may be interested in}

Interfacial band alignment and structural properties of nanoscale $\mathrm{TiO} 2$ thin films for integration with epitaxial crystallographic oriented germanium

J. Appl. Phys. 115, 024303 (2014); 10.1063/1.4861137

Heterojunction band offsets and dipole formation at $\mathrm{BaTiO} / \mathrm{SrTiO} 3$ interfaces

J. Appl. Phys. 114, 183701 (2013); 10.1063/1.4829695

Structural and band alignment properties of Al2O3 on epitaxial Ge grown on (100), (110), and (111)A GaAs substrates by molecular beam epitaxy

J. Appl. Phys. 113, 134311 (2013); 10.1063/1.4799367

Energy band alignment of atomic layer deposited $\mathrm{HfO} 2$ on epitaxial (110)Ge grown by molecular beam epitaxy Appl. Phys. Lett. 102, 093109 (2013); 10.1063/1.4794838

Band offsets of Er 2 O 3 films epitaxially grown on Si substrates

Appl. Phys. Lett. 88, 162909 (2006); 10.1063/1.2196476

MIT LINCOLN

LABORATORY CAREERS

Discover the satisfaction of innovation and service

to the nation
- Space Control

- Air \& Missile Defense

- Communications Systems \& Cyber Security

- Intelligence, Surveillance and

Reconnaissance Systems

- Advanced
Electronics
- Tactical Systems
- Homeland
Protection
- Air Traffic Control

LINCOLN LABORATORY

MassachusetTs Institute of TeChNOLOGY

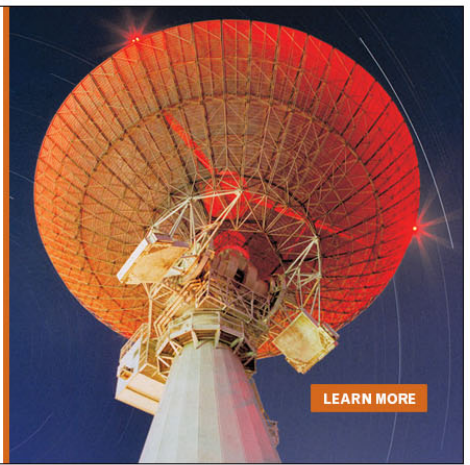




\title{
Quasi-zero lattice mismatch and band alignment of $\mathrm{BaTiO}_{3}$ on epitaxial (110)Ge
}

\author{
M. K. Hudait, ${ }^{1, a)}$ Y. Zhu, ${ }^{1}$ N. Jain, ${ }^{1}$ D. Maurya,${ }^{2}$ Y. Zhou, ${ }^{2}$ and S Priya ${ }^{2}$ \\ ${ }^{1}$ Bradley Department of Electrical and Computer Engineering, Virginia Tech, Blacksburg, Virginia 24061, \\ USA \\ ${ }^{2}$ Center for Energy Harvesting Materials and Systems (CEHMS), Virginia Tech, Blacksburg, Virginia 24061, \\ USA
}

(Received 28 May 2013; accepted 20 June 2013; published online 9 July 2013)

\begin{abstract}
Growth, structural, and band alignment properties of pulsed laser deposited amorphous $\mathrm{BaTiO}_{3}$ on epitaxial molecular beam epitaxy grown (110)Ge layer, as well as their utilization in low power transistor are reported. High-resolution $\mathrm{x}$-ray diffraction demonstrated quasi-zero lattice mismatch of $\mathrm{BaTiO}_{3}$ on $(110) \mathrm{Ge}$. Cross-sectional transmission electron microscopy micrograph confirms the amorphous nature of $\mathrm{BaTiO}_{3}$ layer as well as shows a sharp heterointerface between $\mathrm{BaTiO}_{3}$ and $\mathrm{Ge}$ with no traceable interfacial layer. The valence band offset, $\Delta \mathrm{E}_{\mathrm{v}}$, of $1.99 \pm 0.05 \mathrm{eV}$ at the $\mathrm{BaTiO}_{3} /(110) \mathrm{Ge}$ heterointerface is measured using x-ray photoelectron spectroscopy. The conduction band offset, $\Delta \mathrm{E}_{\mathrm{c}}$, of $1.14 \pm 0.1 \mathrm{eV}$ is calculated using the bandgap energies of $\mathrm{BaTiO}_{3}$ of $3.8 \mathrm{eV}$ and $\mathrm{Ge}$ of $0.67 \mathrm{eV}$. These band offset parameters for carrier confinement and the interface chemical properties of the $\mathrm{BaTiO}_{3} /(110) \mathrm{Ge}$ system are significant advancement towards designing Ge-based p-and n-channel metal-oxide semiconductor field-effect transistors for low-power application. (C) 2013 AIP Publishing LLC. [http://dx.doi.org/10.1063/1.4813226]
\end{abstract}

\section{INTRODUCTION}

Shrinking feature sizes of silicon complementary metaloxide semiconductor (CMOS) transistor have enabled increase in transistor density. This rising number of transistors increases the power consumption in integrated circuits and also constrains the high-speed operation. Low bandgap channel material with superior transport properties and high- $\kappa$ gate dielectric are required to overcome this limitation and achieve further increase in transistor drive current and minimize the gate leakage. In the post-Si CMOS era, germanium (Ge) would be a potential candidate due to its higher electron and hole mobilities which will enhance the carrier transport properties. Compared with $\mathrm{Si}$, Ge has low field bulk mobility gains up to $2 \times$ for electrons and $4 \times$ for holes. Moreover, Ge is more compatible than $\mathrm{Si}$ with the most promising recent high- $\kappa$ gate dielectrics ${ }^{1}$ which eliminates the unwanted low $\kappa$ interfacial dielectric layer normally formed on Si. Furthermore, low bandgap Ge is compliant with the requirement of lower supply voltage operation of transistor. In fact, Ge is an excellent template for III-V heteroepitaxy and can be heterogeneously integrated on $\mathrm{Si}$ in conjunction with various optoelectronic components that could allow extending the Moore's law. ${ }^{2}$

Very recently, it has been demonstrated that the carrier mobility of Ge metal-oxide semiconductor field effect transistors (MOSFETs) can be enhanced by utilizing a Ge channel with different orientations. The carrier mobility was expected to be high in (111)Ge for electrons ${ }^{3}$ and in $(110) \mathrm{Ge}$ for holes. ${ }^{4}$ Recently, it has been demonstrated that the transistors fabricated on (110)Ge substrate exhibited higher hole mobilities of $650 \mathrm{~cm}^{2} / \mathrm{V} \mathrm{s}$ (Ref. 5) and higher electron mobility in (111)Ge

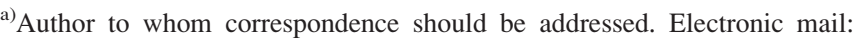
mantu.hudait@vt.edu. Tel.: (540) 231-6663. Fax: (540) 231-3362.
}

orientation as compared to that of $(100) \mathrm{Ge}^{6}{ }^{6}$ Dissanayake et al. ${ }^{7}$ have reported that the hole mobility of (110)Ge channel oriented along the $\langle 110\rangle$ direction exhibited $2.3 \times$ higher hole mobility compared with the (100) Ge surface. According to Yang et al., ${ }^{8}$ the electron mobility of Ge with (111) orientation is $1.8 \times$ higher than the electron mobility of Ge with (100) and (110) orientations. These crystallographic oriented epitaxial Ge layers were demonstrated on GaAs and the detailed band offset properties of the crystallographically oriented $\mathrm{GaAs} / \mathrm{Ge} / \mathrm{GaAs}$ heterostructure have been reported by Hudait et al. $^{9}$ These advancements have intensified the research on Ge integration with the possibility of exceeding Moore's law.

Significant research has been conducted on the high- $\kappa$ gate dielectrics such as $\mathrm{HfO}_{2}, \mathrm{ZrO}_{2}$, and $\mathrm{Al}_{2} \mathrm{O}_{3}$ grown on (100)Ge, ${ }^{10-18}$ (110)Ge, ${ }^{1,15,16}$ and (111)Ge. ${ }^{15,16}$ Among the new high- $\kappa$ dielectric materials, $\mathrm{BaTiO}_{3}$ (BTO) directly grown on semiconductors has attracted attention for nonvolatile single transistor memory, ${ }^{19}$ to reduce the susbthreshold swing and increase the on-current of a transistor. ${ }^{20,21}$ BTO has also been used as a buffer layer for integration of $\mathrm{Ge}$ on $\mathrm{Si}$ and continues to be of interest for added functionalities on $\mathrm{Si}$ or $\mathrm{Ge}$ for low-power CMOS logic applications. However, integration of BTO on semiconductors faces numerous challenges such as: (i) the epitaxial nucleation requires oxide formation on the surface, ${ }^{22}$ (ii) requirement of oxide buffer layer (e.g., $\mathrm{BaSrO}, \mathrm{BaO}, \mathrm{MgO}, \mathrm{SrTiO}_{3}$ (STO)) between the BTO and the parent substrate to ensure that BTO is $c$-axis oriented, ${ }^{22-27}$ (iii) monolayer (ML) of strontium ( $\mathrm{Sr}$ ) metal as the nucleation layer, ${ }^{28}$ and (iv) large lattice mismatch $(>20 \%)$ with Ge-on-Si(001). ${ }^{29}$ Vaithyanathan et al. ${ }^{30}$ suggest that a thick buffer layer of $\mathrm{BaSrO}$ is needed for $c$-axis oriented BTO when grown on $\mathrm{Si}$. In their study, using pulsed laser deposition (PLD), a preferential $c$-axis 
oriented BTO was deposited on a $\mathrm{MgO}$ buffer layer that was grown on GaAs by molecular beam epitaxy $(\mathrm{MBE})^{31}$ and PLD. ${ }^{32}$ Huang et al. ${ }^{33}$ demonstrated columnar nature of BTO film on GaAs using STO buffer using PLD and the crystal quality of the BTO film was found to be strongly dependent on the quality of the STO buffer layer. However, amorphous and the high dielectric constant BTO with abrupt interface between the BTO and the channel layer is necessary to reduce the gate leakage in a transistor.

It is well-known that BTO has a tetragonal crystal structure at room temperature with $a$ - and $b$-axis lattice constant of $3.992 \AA$ and $c$-axis lattice constant of $4.036 \AA$. Unless, the BTO lattice is rotated by $45^{\circ}$ (c-axis oriented) with respect to the $(001) \mathrm{Ge}$ or $(001) \mathrm{GaAs}\left(\mathrm{a}_{\mathrm{Ge}(\mathrm{GaAs})} \approx 5.6543 \AA\right)$, the lattice mismatch is greater than $20 \%$. It is limited to $<0.2 \%$ lattice mismatch if the BTO growth aligns with in-plane lattice constant of (100)Ge or (100)GaAs. In order to achieve the rotation of the BTO lattice, several approaches have been proposed, namely, deposition of ultra-thin barium (Ba) interlayer, ${ }^{29} \mathrm{BaO}$ interlayer, ${ }^{23}$ STO nucleation layer, ${ }^{22,33}$ and $\mathrm{MgO}$ buffer layer. ${ }^{31,32}$ A robust quasi-zero lattice mismatched BTO growth on oriented Ge layer is highly preferred without any interlayer to achieve desired electrical characteristics and to make the process compatible with standard fabrication.

In this study, we demonstrate the growth and band alignment properties of pulsed laser deposited BTO on epitaxial MBE grown (110)Ge layer. Compared with the other approaches mentioned above, quasi-zero lattice mismatch was found to exist between BTO and the (110)Ge layer without any need for intermediate interlayer. Our results provide a significant advancement towards the control of the strainfree BTO films on semiconductor surface. High-quality BTO with high- $\kappa$ on $(110) \mathrm{Ge}$ interface is expected to provide desired valence and conduction band discontinuities, $\Delta \mathrm{E}_{\mathrm{v}}$ and $\Delta \mathrm{E}_{\mathrm{c}}$ larger than $1 \mathrm{eV}$ relative to the $(110) \mathrm{Ge}$ channel material. ${ }^{34}$ These discontinuities are essential to act as a barrier for both holes and electrons. The experimental results from this study are the first step towards achieving highperformance metal/BTO/(110)Ge metal-oxide semiconductor (MOS) capacitor that can be used for the p-channel Ge based MOSFET applications.

\section{EXPERIMENTAL}

\section{A. Perovskite film deposition}

The undoped epitaxial $80 \mathrm{~nm}$ thick (110)Ge layers were grown using in-situ growth process on epi-ready (110)GaAs substrates using separate solid source MBE growth chambers for Ge and III-V materials, connected via ultra-high vacuum transfer chamber. The growth temperature and growth rate of Ge were $400^{\circ} \mathrm{C}$ and $0.1 \AA / s$, respectively. The details of the growth procedure are reported elsewhere., ${ }^{9,35}$ The $1 \mathrm{~nm}$ and $10 \mathrm{~nm}$ BTO films were grown by PLD using a $\mathrm{KrF}$ excimer laser $(\lambda=248 \mathrm{~nm})$ on epitaxial $(110) \mathrm{Ge}$ at a deposition rate of $\sim 0.5 \AA$ As. Stoichiometric BTO target was synthesized by conventional mixed-oxide processing route. The focused laser beam irradiates the rotating $(89 \mathrm{rpm})$ target with a laser energy density of $\sim 2.5 \mathrm{~J} / \mathrm{cm}^{2}$ at a repetition rate of $10 \mathrm{~Hz}$. The deposition was made using a vacuum chamber with oxygen pressure of 100 mTorr during the deposition of BTO films on (110)Ge layers. During the BTO growth, the substrate temperature of $(110) \mathrm{Ge}$ film was kept constant at $250^{\circ} \mathrm{C}$. Epitaxial (110)Ge layers were cleaned using $\mathrm{NH}_{4} \mathrm{OH}: \mathrm{H}_{2} \mathrm{O}_{2}: \mathrm{H}_{2} \mathrm{O}$ (2:1:1000 volume ratio) for $5 \mathrm{~s}$ prior loading to PLD chamber for BTO deposition.

\section{B. Characterizations}

To determine the structural quality and the relaxation state of BTO on epitaxial $(110) \mathrm{Ge}$, high-resolution triple axis X-ray rocking curves were recorded. Cross-sectional high-resolution transmission electron microscopy (HR-TEM) was used to characterize the interface between the BTO and the $(110) \mathrm{Ge}$ epilayer. HR-TEM imaging was performed using FEI Titan 80-300 transmission electron microscope. For this purpose, the electron transparent foils of thin film cross-section of $\mathrm{BaTiO}_{3} /$ $\mathrm{Ge} /(110) \mathrm{GaAs}$ were prepared by standard polishing technique, i.e., mechanical grinding, dimpling, and $\mathrm{Ar}^{+}$ion beam milling. The band alignment of BTO/(110)Ge structures was investigated using a PHI Quantera SXM XPS system with a mono-

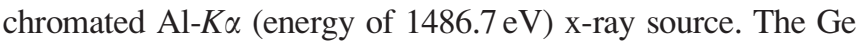
$3 \mathrm{~d}$ and $\mathrm{Ba} 4 \mathrm{~d}_{5 / 2}$ core level (CL) binding energy spectra as well as $\mathrm{Ge}$ and $\mathrm{Ba}$ valence band (VB) binding energy spectra were collected with a pass energy of $26 \mathrm{eV}$ and an exit angle of $45^{\circ}$. The binding energy was corrected by adjusting the carbon $(\mathrm{C})$ 1s CL peak position to $285.0 \mathrm{eV}$ for each sample surface. Curve fitting was done by the CasaXPS 2.3.14 using a Lorentzian convolution with a Shirley-type background. The CL energy position was defined to be the center of the peak width at the half of the peak height. The CL and valence electrons emitted from each film determined from the XPS measurement will allow to determine the $\Delta \mathrm{E}_{\mathrm{v}}$ of $\mathrm{BTO}$ relative to the (110)Ge by the method described in Ref. 36. The error bar we defined in this paper is due to the scatter of valence band spectra during the fitting of valence band maximum (VBM) position and considering the linearity and stability of the energy scale of the XPS binding energy spectrum. The VBM values are determined by linear extrapolation of the leading edge to the base line of the VB spectra recorded on the $10 \mathrm{~nm}$ BTO and $80 \mathrm{~nm}$ Ge film to the base lines. Indeed, the VBM value is sensitive to the choice of points on the leading edge used to obtain the regression line. ${ }^{37}$ Several different sets of points were selected over the linear region of the leading edge to perform regressions, and the uncertainty of $\Delta \mathrm{E}_{\mathrm{v}}$ and $\Delta \mathrm{E}_{\mathrm{c}}$ values was found to be in the range of $0.05-0.1 \mathrm{eV}$ in the present work.

\section{RESULTS AND DISCUSSION}

\section{A. X-ray rocking curves of $\mathrm{BaTiO}_{3}$ on $(110) \mathrm{Ge}$}

Figure 1(a) shows the high-resolution triple axis symmetric X-ray rocking curve from the (002) Bragg line of $10 \mathrm{nmBTO} / 80 \mathrm{~nm}(110) \mathrm{Ge} /(110) \mathrm{GaAs}$ structure, where the epitaxial Ge layer thickness is significantly lower than the critical layer thickness. The significantly small angular separation between the (002) diffraction peaks of BTO/(110)Ge and the (110)GaAs substrate resulting from the difference in 

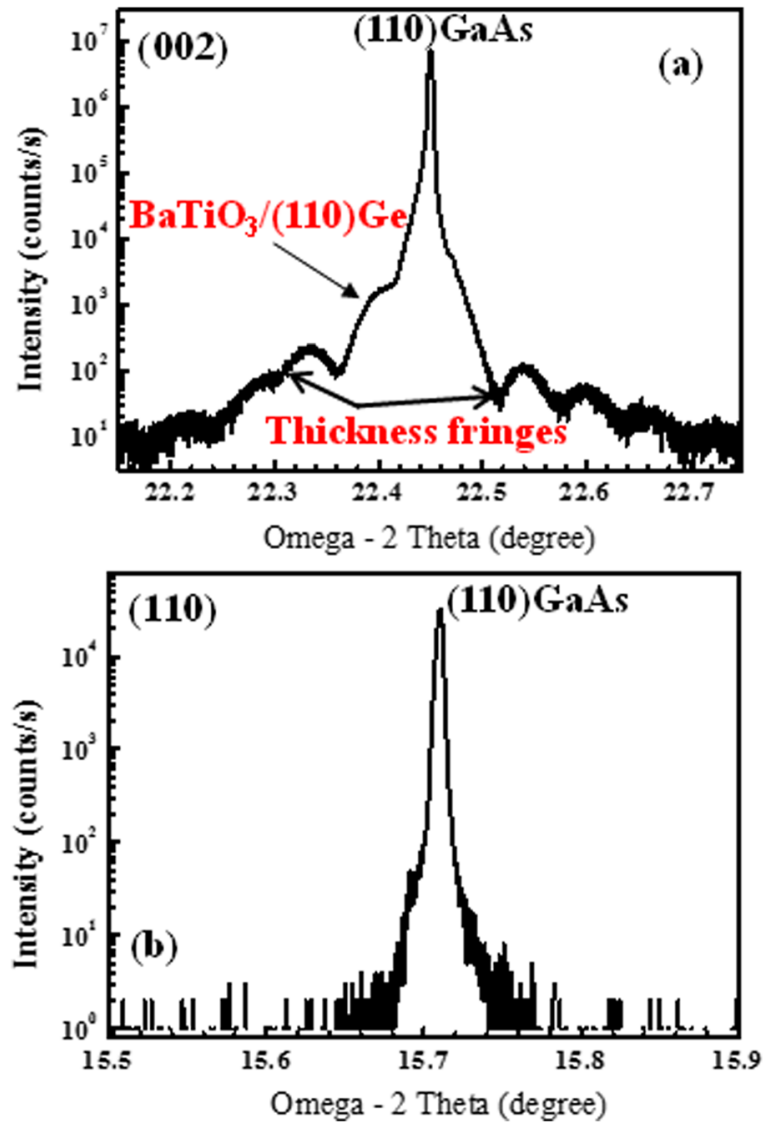

FIG. 1. X-ray rocking curves from (a) symmetric (002) and (b) asymmetric (110) reflection of $10 \mathrm{~nm} \mathrm{BaTiO}_{3} / 80 \mathrm{~nm}$ (110)Ge structure on (110)GaAs substrate, respectively. The Pendellösung oscillations in the rocking curve confirm the high crystalline quality of the $\mathrm{BaTiO}_{3} / \mathrm{Ge} /(110) \mathrm{GaAs}$ interface.

lattice plane spacing indicates the perfect lattice matched layers of BTO, Ge, and GaAs. The appearance of strong Pendellösung oscillation fringes on both sides of $\mathrm{BTO} / \mathrm{Ge}$ and GaAs peaks implies the presence of sharp heterointerfaces, similar to the lattice matched $\mathrm{GaAs} / \mathrm{Ge} / \mathrm{GaAs}$ heterostructure recently reported in Ref. 9. These fringes originate from the beating of two $\mathrm{x}$-ray wave fields inside the structure. One of the wave field is generated at the interface between the GaAs and $\mathrm{Ge}$ and another wave field at the interface of BTO/(110)Ge. As a result, interference can only be observed in a structure that has almost perfectly parallel boundaries. Figure 1(b) shows the X-ray rocking curve from asymmetric (110) Bragg line of BTO/(110)Ge/(110)GaAs structure. The relatively narrow full width at half maximum of the (110) x-ray peak indicates the high-quality of the structure. Merckling et al. ${ }^{29}$ found two distinct BTO phases for the films grown on $\mathrm{Ge} /(100) \mathrm{Si}$. The tetragonal BTO phase was strained on the (100)Ge substrate and the remaining BTO film had a cubic symmetry. However, the combined effect of the high temperature Ge oxide desorption at $800^{\circ} \mathrm{C}$ for $30 \mathrm{~min}$ and the $650^{\circ} \mathrm{C}$ growth temperature required for $\mathrm{BTO}$ during the MBE growth process led to crystallographic disorder at the $\mathrm{BTO} /(100) \mathrm{Ge}$ interface. Contreras-Guerrero et $a l^{22}$ demonstrated MBE grown epitaxial BTO on (100)GaAs with $8 \AA$ STO buffer layer. In their study, it was hypothesized that the BTO layer is rotated by $45^{\circ}$ with respect to GaAs layer suggesting that the BTO (110) planes are parallel to the GaAs (100) planes. However, there were no HR-TEM results included to support the hypothesis.

\section{B. Cross-sectional TEM micrographs of $\mathrm{BaTiO}_{3}$ on (110)Ge}

Figures 2(a) and 2(b) show the cross-sectional transmission electron microscopy (TEM) micrograph of BTO/ $(110) \mathrm{Ge} /(110) \mathrm{GaAs}$ interface and high-resolution TEM micrograph of BTO/(110)Ge interface, respectively. From these micrographs, we can clearly observe sharp interface of BTO and Ge. The TEM results show that the pulse laser deposited $\sim 10 \mathrm{~nm}$ thick amorphous BTO layer were grown with high degree of coherency on (110)Ge. From Fig. 2(b), one can find that there was no interfacial layer $(\mathrm{GeO}$ and $\mathrm{GeO}_{2}$ (Refs. 38 and 39)) formed during the deposition of BTO on (110)Ge layer which implies that the removal of interfacial oxide can be easily achieved on (110)Ge. This is a significant potential advantage of higher- $\kappa$ BTO on (110)Ge for low-power transistor application. The coherency and composition at the interface were confirmed through elemental analysis and high resolution lattice mapping. In conjunction with the results from $x$-ray diffraction, TEM analysis confirmed the strain-free BTO growth on the (110) Ge.

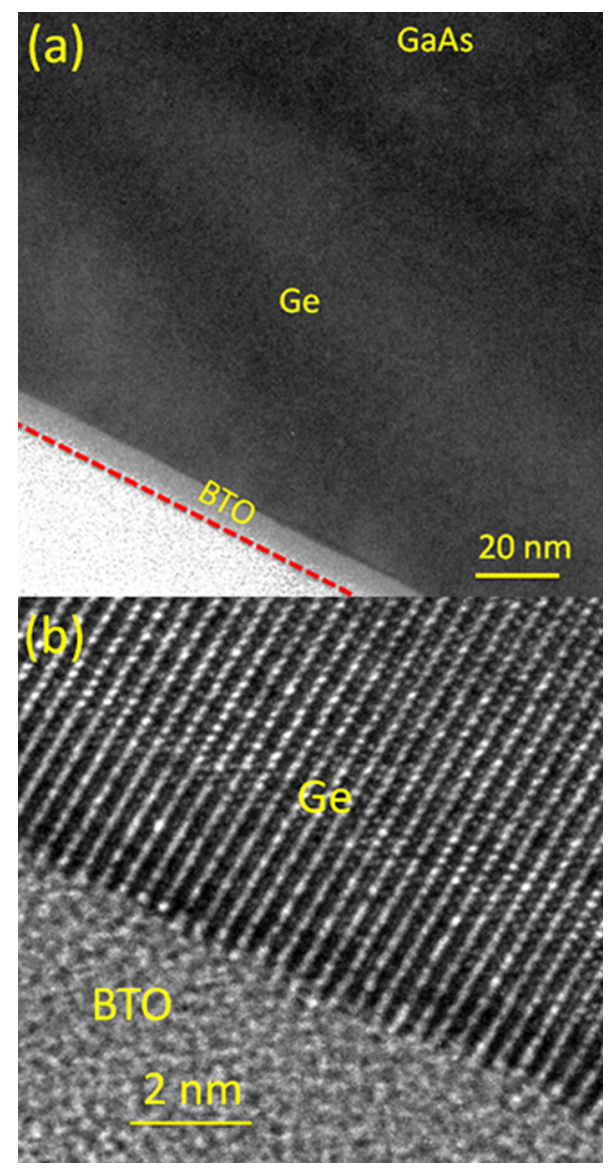

FIG. 2. (a) Cross-sectional TEM micrograph of $\mathrm{BaTiO}_{3}$ layer deposited on epitaxial Ge grown on (110) GaAs substrate. (b) High-resolution TEM micrograph at the $\mathrm{BaTiO}_{3} /(110) \mathrm{Ge}$ interface. 


\section{Energy band alignment of $\mathrm{BaTiO}_{3}$ on (110)Ge}

The energy band alignment at the $\mathrm{BTO} / \mathrm{Ge}$ interface is critical since the sufficient barrier for electron and hole is needed to suppress the tunneling leakage current. The valence band offset, $\Delta \mathrm{E}_{\mathrm{v}}$ at the $\mathrm{BTO} /(110) \mathrm{Ge}$ was determined using XPS system and angle integrated photoelectron energy distribution curves for the VBM. Using these methods, Ge $3 \mathrm{~d}$ and $\mathrm{Ba} 4 \mathrm{~d}_{5 / 2} \mathrm{CL}$ spectra were recorded. The peak separation of Ge $3 d_{5 / 2}$ and $3 d_{3 / 2}$ due to spin-orbit splitting was too small to be separated. The binding energy was corrected by adjusting the $\mathrm{C} 1 \mathrm{~s}$ core-level peak position to $285.0 \mathrm{eV}$ for each sample surface. XPS spectra were recorded from the following 3 samples: (i) (110)Ge epitaxial layer, (ii) $1 \mathrm{~nm}$ BTO on (110)Ge layer, and (iii) $10 \mathrm{~nm}$ thick BTO film on $(110) \mathrm{Ge}$. Figure 3 shows the XPS spectra of Ge $3 \mathrm{~d}$ core level $\left(E_{G e 3 d}^{G e}\right)$ and the VBM $\left(E_{V B M}^{G e}\right)$ of the $(110)$ Ge film, respectively. Figure 4 shows XPS spectra of (a) $\mathrm{Ba} 4 \mathrm{~d}_{5 / 2}\left(E_{B a 4 d_{5 / 2}}^{B a}\right)$ core level and (b) VBM $\left(E_{V B M}^{B a}\right)$ from $10 \mathrm{~nm}$ BTO film, respectively. Figure 5 shows the (a) (110)Ge $3 \mathrm{~d}$ core level $\left(E_{G e 3 d}^{G e}\right)$ and $\mathrm{Ba} 4 \mathrm{~d}_{5 / 2}$ core level $\left(E_{B a 4 d_{5 / 2}}^{B a}\right)$ spectrum of $1 \mathrm{~nm}$ BTO on (110)Ge interface, respectively. The valence band offset, $\Delta \mathrm{E}_{\mathrm{v}}$ for a $\mathrm{BTO} /(110) \mathrm{Ge}$ interface was determined from the following equation ${ }^{36}$ using $\mathrm{CL}$ spectra:
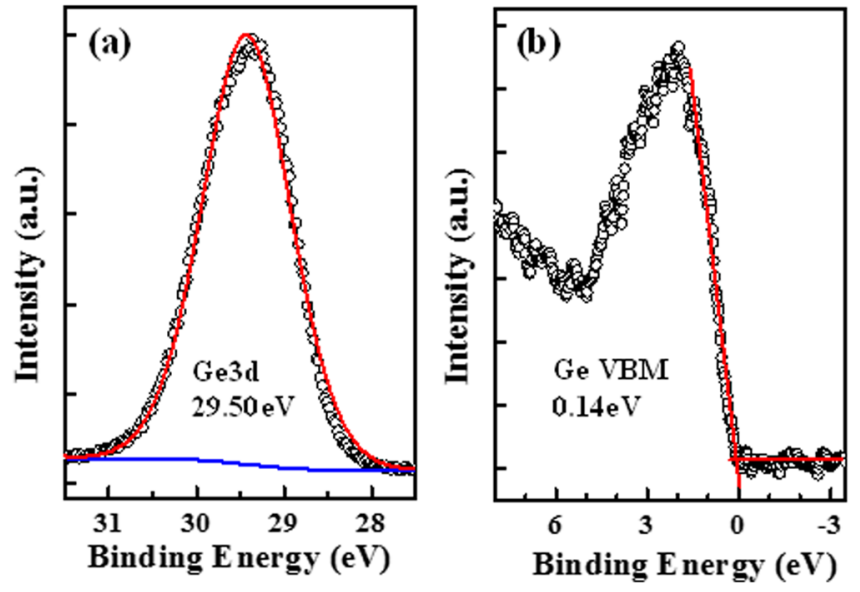

FIG. 3. XPS spectra of (a) Ge $3 \mathrm{~d}$ core level $\left(E_{G e 3 d}^{G e}\right)$ and (b) valence band maximum, VBM $\left(E_{V B M}^{G e}\right)$ from epitaxial $(110) \mathrm{Ge}$ film, respectively.
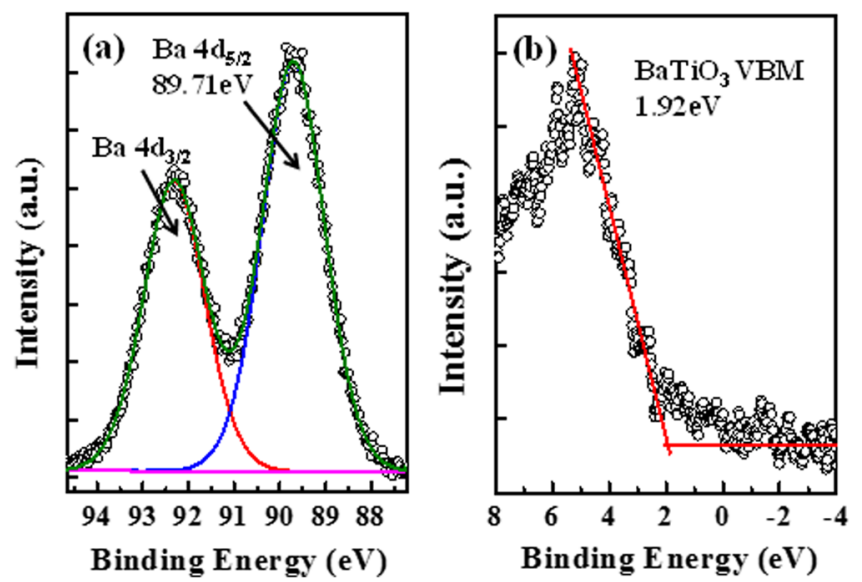

FIG. 4. XPS spectra of (a) Ba $4 \mathrm{~d}_{5 / 2}\left(E_{B a 4 d_{5 / 2}}^{B a}\right)$ core level and (b) VBM $\left(E_{V B M}^{B a}\right)$ from $10 \mathrm{~nm} \mathrm{BaTiO}_{3}$ film, respectively.
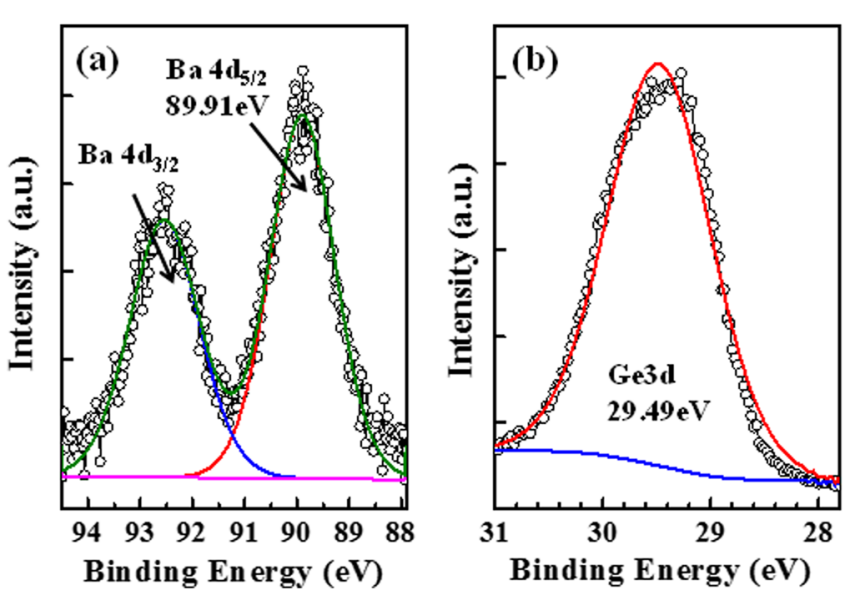

FIG. 5. XPS spectra of (a) Ba $4 \mathrm{~d}_{5 / 2}\left(E_{B a 4 d_{5 / 2}}^{B a}\right)$ and (b) $\mathrm{Ge} 3 \mathrm{~d}\left(E_{G e 3 d}^{G e}\right)$ core levels from $1 \mathrm{~nm}$ thin $\mathrm{BaTiO}_{3}$ film/(110)Ge interface.

$$
\begin{aligned}
\Delta E_{V}= & \left(E_{G e 3 d}^{G e}-E_{V B M}^{G e}\right)^{(110) G e}-\left(E_{B a 4 d_{5 / 2}}^{B a}-E_{V B M}^{B a}\right)^{10 n m B T O} \\
& -\left(E_{G e 3 d}^{G e}-E_{B a 4 d_{5 / 2}}^{B a}\right)^{1 n m B T O /(110) G e \text { interface }}
\end{aligned}
$$

The Ba $4 d_{5 / 2}$ CL spectra were selected rather than $B a 4 d_{3 / 2}$ since the measured binding energy separation between the $\mathrm{Ba} 4 \mathrm{~d}_{5 / 2}$ and $\mathrm{Ba} 4 \mathrm{~d}_{3 / 2}$ peaks was fixed. As a result, the band offset result would not change if $B a d_{3 / 2}$ was selected as the CL binding energy peak. Finally, the conduction band offset, $\Delta \mathrm{E}_{\mathrm{c}}$, for a $\mathrm{BTO} /(110) \mathrm{Ge}$ interface was determined from the following equation, $\Delta E_{C}=E_{g}^{B T O}-E_{g}^{G e}-\Delta E_{V}$, where $E_{g}^{B T O}$ and $E_{g}^{G e}$ are the bandgaps of BTO and $\mathrm{Ge}$, respectively.

The position of the (110)Ge 3d peak centroid from the XPS measurement was found to be $30.05 \pm 0.05 \mathrm{eV}$ as shown in Fig. 3. This value was obtained by measuring the center of the peak width at half of the peak height after Shirley background subtraction. ${ }^{10}$ The VBM for $(110) \mathrm{Ge}$ was determined as the intersection between the linear fits of the background and the linear portion of the VB leading edge, ${ }^{10}$ as shown in the inset of Fig. 3. The energy difference between the Ge $3 \mathrm{~d}$ centroid and the (110)Ge VBM was measured to be $29.36 \pm 0.05 \mathrm{eV}$. Similarly, the energy difference between the Ba $4 \mathrm{~d}_{5 / 2}$ centroid and the VBM was found to be $87.79 \pm 0.05 \mathrm{eV}$ for the $10 \mathrm{~nm}$ thick BTO film. For the $1 \mathrm{~nm}$ BTO film on (110)Ge, the energy difference between the $\mathrm{Ge} 3 \mathrm{~d}$ centroid and the $\mathrm{Ba} 4 \mathrm{~d}_{5 / 2}$ CLs was determined to be $60.42 \pm 0.05 \mathrm{eV}$. Using these measured data and Eq. (1), the measured value of $\Delta \mathrm{E}_{\mathrm{v}}$ for the $\mathrm{BTO} /(110) \mathrm{Ge}$ interface was found to be $1.99 \pm 0.05 \mathrm{eV}$ and this value was lower by $\sim 0.6 \mathrm{eV}$ than that for $\mathrm{BTO} /(100) \mathrm{Ge}$ substrate, ${ }^{28}$ where $0.5 \mathrm{ML}$ of $\mathrm{Sr}$ metal was used as a nucleation layer. It was lower by $\sim 0.8 \mathrm{eV},{ }^{23}$ in comparison to the substrate where 1 atomic plane of $\mathrm{BaO}$ layer was used as an interlayer between BTO and (100)Ge. The observed differences in the $\Delta \mathrm{E}_{\mathrm{v}}$ values for the BTO on $(110) \mathrm{Ge}$ in this work and BTO on $(100) \mathrm{Ge}$ with interlayers by other researchers ${ }^{23,28}$ can be explained due to the difference in surface reconstruction of the (100)Ge and (110)Ge layers on GaAs substrates. ${ }^{9}$ The differences present in surface structure of Ge (i.e., $(2 \times 1)$, $(2 \times 2),(3 \times 4))$ would influence the band offset of BTO on 
crystallographically oriented epitaxial Ge resulting in variation in the values of band offsets. The deposition temperature of BTO on (110)Ge layer during PLD process was lower than the crystallization temperature of BTO resulting in the formation of amorphous layer. Thus, any dangling bonds at the surface of (110) Ge were able to accommodate the oxygen atoms during deposition of BTO. However, there is no evidence of interfacial $\mathrm{GeOx}$ layer in the TEM images shown in Fig. 2. This suggests that a robust interface prevents the oxidation of the (110)Ge surface similar to the case of $\mathrm{HfO}_{2}$ on $(110) \mathrm{Ge} .{ }^{1}$ It has been reported that band offsets in addition to substrate orientation and surface structure can also depend on various other parameters such as overlayer crystallinity, deposition temperature, deposition rate, microscopic interface dipole and interdiffusion or reactivity. ${ }^{40}$ However, in the case of amorphous BTO/(110)Ge, the surface reconstruction has the dominant effect as compared to the other variables. The $\Delta \mathrm{E}_{\mathrm{c}}$ for the $\mathrm{BTO}$ on $(110) \mathrm{Ge}$ was calculated to be $1.14 \pm 0.1 \mathrm{eV}$ using the $3.8 \pm 0.1 \mathrm{eV}$ bandgap of thin BTO layer ${ }^{41}$ and $0.67 \mathrm{eV}$ bandgap of Ge. Figure 6 shows the band alignment diagram of the BTO/(110) Ge heterojunction based on XPS results. Both $\Delta \mathrm{E}_{\mathrm{v}}$ and $\Delta \mathrm{E}_{\mathrm{c}}$ are $1 \mathrm{eV}$, as needed for blocking the electrons and holes ${ }^{34}$ for carrier transport in Ge MOSFETs.

The determination of the band offset between semiconductor/semiconductor heterojunction was developed by Kraut et $a l .^{36}$ using XPS technique and currently this method is widely used to determine the valence band offset between dielectric and semiconductor heterojunction. $1,10,13,15,16,42-44$ However, the measures must be taken in order to compensate the positive changes generated during XPS measurement. ${ }^{42-45}$ Unless, these positive charges were neutralized during the XPS measurement by flowing electron through the sample, there could be positive charge accumulation over the surface and potentially affect the band bending due to insufficient compensation of electron loss. In this differential charging effect, the photoelectrons emitted from semiconductor was compensated by flowing electron through the sample holder

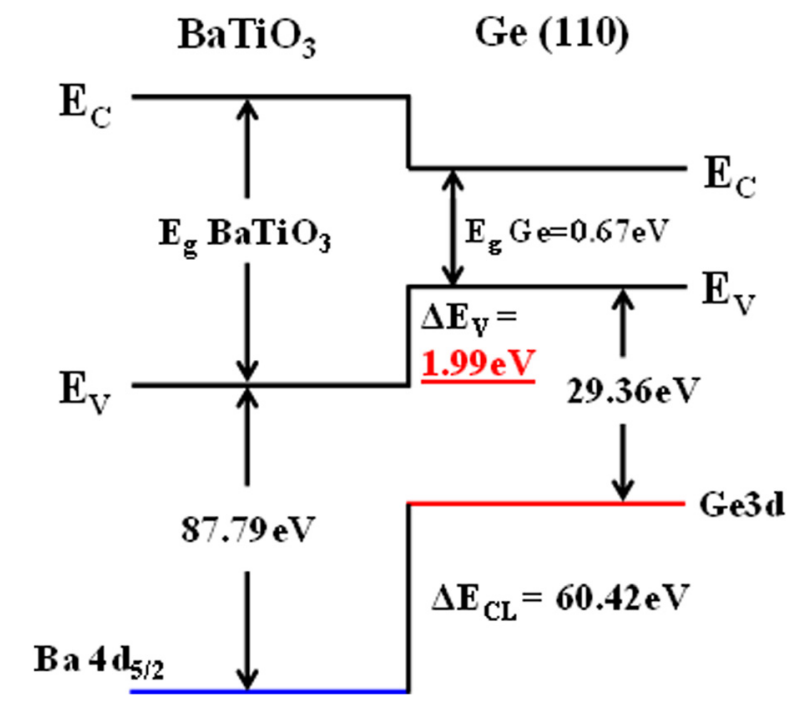

FIG. 6. Energy-band diagram of the $\mathrm{BaTiO}_{3} /(110) \mathrm{Ge}$ heterojunction obtained from XPS measurements. and may not be fully neutralized from the dielectric material due to insulating nature of the film that could modify the core level signals from the dielectric and subsequently affect the valence band offset. ${ }^{42-44}$ During our XPS measurement, we have used continuous flow of electron to minimize the binding energy shift caused by the charging effect. Also, the CL and VB were shifted by the same amount such that the difference between these binding energy values has a less significant effect on the valence band offset of BTO on Ge. Besides, all measured CLs and valence band binding energy values were corrected by shifting C $1 \mathrm{~s}$ core level peak to $285.0 \mathrm{eV}$. Thus, the influence of charging effect on the measured valence band offset between BTO and (110)Ge is minimized.

\section{CONCLUSIONS}

In summary, pulsed laser deposited $\mathrm{BaTiO}_{3}$ layers with quasi-zero lattice mismatch on epitaxial (110)Ge were demonstrated by high-resolution x-ray diffraction. Cross-sectional TEM micrography confirmed that amorphous BTO layer on (110)Ge showed a sharp interface. There was no trace of interfacial layer between the BTO film and the (110)Ge epilayer. XPS results showed the $\Delta \mathrm{E}_{\mathrm{v}}$ of $1.99 \pm 0.05 \mathrm{eV}$ at the BTO/ (110)Ge heterointerface. The $\Delta \mathrm{E}_{\mathrm{c}}$ was calculated to be $1.14 \pm 0.1 \mathrm{eV}$ using the bandgap of BTO of $3.8 \mathrm{eV}$ and with the Ge bandgap of $0.67 \mathrm{eV}$. These band offset parameters for carrier confinement and the interface chemical properties of the $\mathrm{BaTiO}_{3} /(110) \mathrm{Ge}$ system are significant advancements towards designing Ge-based p-and n-channel MOSFETs for low-power application.

\section{ACKNOWLEDGMENTS}

The part of the work was supported by Intel Corporation. S.P. and D.M. would like to acknowledge the financial support from Office of Basic Science, Department of Energy, through Grant No. DE-FG02-06ER46290.

${ }^{1}$ M. K. Hudait, Y. Zhu, D. Maurya, and S. Priya, Appl. Phys. Lett. 102, 93109 (2013).

${ }^{2}$ International Technology Roadmap for Semiconductors (ITRS), Process Integration, Devices and Structures (PIDS), 2011 ed. (ITRS, 2011).

${ }^{3}$ K. Minami, Y. Nakamura, S. Yamasaka, O. Yoshitake, J. Kikkawa, K. Izunome, and A. Sakai, Thin Solid Films 520, 3232 (2012).

${ }^{4}$ T. Krishnamohan, D. Kim, T. V. Dinh, A. Pham, B. Meinerzhagen, C. Jungemann, and K. Saraswat, in IEEE Conference Proceedings of International Electron Devices Meeting (IEDM) (IEEE, New York, 2008), p. 899.

${ }^{5}$ B.-F. Hsieh and S.-T. Chang, Solid-State Electron. 60, 37 (2011).

${ }^{6}$ C. H. Lee, T. Nishimura, N. Saido, K. Nagashio, K. Kita, and A. Toriumi, in IEEE Conference Proceedings of International Electron Devices Meeting (IEDM) (IEEE, New York, 2009), p. 457.

${ }^{7}$ S. Dissanayake, Y. Zhao, S. Sugahara, M. Takenaka, and S. Takaghi, J. Appl. Phys. 109, 033709 (2011).

${ }^{8}$ Y. J. Yang, W. S. Ho, C. F. Haung, S. T. Chang, and C. W. Liu, Appl. Phys. Lett. 91, 102103 (2007).

${ }^{9}$ M. K. Hudait, Y. Zhu, N. Jain, and J. L. Hunter, Jr., J. Vac. Sci. Technol. B 31, 11206 (2013).

${ }^{10}$ M. Perego, G. Seguini, and M. Fanciulli, J. Appl. Phys. 100, 93718 (2006).

${ }^{11}$ S. J. Wang, A. C. H. Huan, Y. L. Foo, J. W. Chai, J. S. Pan, Q. Li, Y. F. Dong, Y. P. Feng, and C. K. Ong, Appl. Phys. Lett. 85, 4418 (2004).

${ }^{12}$ H. Seo, F. Bellenger, K. B. Chung, M. Houssa, M. Meuris, M. Heyns, and G. Lucovsky, J. Appl. Phys. 106, 44909 (2009). 
${ }^{13}$ S. Swaminathan, Y. Sun, P. Pianetta, and P. C. McIntyre, J. Appl. Phys. 110, 94105 (2011).

${ }^{14}$ J. H. Choi, Y. Mao, and J. P. Chang, Mater. Sci. Eng. R. 72, 97 (2011).

${ }^{15}$ M. K. Hudait and Y. Zhu, J. Appl. Phys. 113, 114303 (2013).

${ }^{16}$ M. K. Hudait, Y. Zhu, D. Maurya, S. Priya, P. K. Patra, A. W. K. Ma, A. Aphale, and I. Macwan, J. Appl. Phys. 113, 134311 (2013).

${ }^{17}$ P. Tsipas and A. Dimoulas, Appl. Phys. Lett. 94, 012114 (2009).

${ }^{18}$ A. Dimoulas, P. Tsipas, A. Sotiropoulos, and E. K. Evangelou, Appl. Phys. Lett. 89, 252110 (2006).

${ }^{19}$ J. Scott, Ferroelectric Memories (Springer, Berlin, 2000).

${ }^{20}$ S. Salahuddin and S. Datta, Nano Lett. 8, 405 (2008).

${ }^{21}$ A. I. Khan, C. W. Yeung, C. Hu, and S. Salahuddin, in IEEE Conference Proceedings of International Electron Devices Meeting (IEDM) (IEEE, New York, 2011), p. 255.

${ }^{22}$ R. Contreras-Guerrero, J. P. Veazey, J. Levy, and R. Droopad, Appl. Phys. Lett. 102, 12907 (2013).

${ }^{23}$ R. A. McKee, F. J. Walker, and M. F. Chisholm, Science 293, 468 (2001).

${ }^{24} \mathrm{H}$. Li, X. Hu, Y. Wei, Z. Yu, X. Zhang, R. Droopad, A. A. Demkov, J. Edwards, K. Moore, W. Ooms, J. Kulik, and P. Fejes, J. Appl. Phys. 93, 4521 (2003).

${ }^{25}$ Y. Liang, J. Kulik, T. Eschrich, R. Droopad, Z. Yu, and P. Maniar, Appl. Phys. Lett. 85, 1217 (2004).

${ }^{26}$ R. A. McKee, F. J. Walker, J. R. Conner, E. D. Specht, and D. E. Zelmon, Appl. Phys. Lett. 59, 782 (1991).

${ }^{27}$ Z. Yu, J. Ramdani, J. A. Curless, C. D. Overgaard, J. M. Finder, R. Droopad, K. W. Eisenbeiser, J. A. Hallmark, W. J. Ooms, and V. S. Kaushik, J. Vac. Sci. Technol. B 18, 2139 (2000).

${ }^{28}$ P. Ponath, A. Posadas, K. Fredrickson, A. Kvit, and A. Demkov, in The American Physical Society March Meeting 2013, Baltimore, MD, 18-22 March 2013.

${ }^{29}$ C. Merckling, G. Saint-Girons, C. Botella, G. Hollinger, M. Heyns, J. Dekoster, and M. Caymax, Appl. Phys. Lett. 98, 92901 (2011).
${ }^{30}$ V. Vaithyanathan, J. Lettieri, W. Tian, A. Sharan, A. Vasudevarao, Y. L. Li, A. Kochhar, H. Ma, J. Levy, P. Zschack, J. C. Woicik, L. Q. Chen, V. Gopalan, and D. G. Schlom, J. Appl. Phys. 100, 24108 (2006).

${ }^{31}$ T. E. Murphy, D. Chen, and J. D. Phillips, Appl. Phys. Lett. 85, 3208 (2004).

${ }^{32}$ D. Chen, T. E. Murphy, S. Chakrabarti, and J. D. Phillips, Appl. Phys. Lett. 85, 5206 (2004).

${ }^{33}$ W. Huang, Z. P. Wu, and J. H. Hao, Appl. Phys. Lett. 94, 032905 (2009).

${ }^{34}$ J. Robertson and B. Falabretti, J. Appl. Phys. 100, 14111 (2006).

${ }^{35}$ M. K. Hudait, Y. Zhu, N. Jain, S. Vijayaraghavan, A. Saha, T. Merritt, and G. A. Khodaparast, J. Vac. Sci. Technol. B 30, 51205 (2012).

${ }^{36}$ E. A. Kraut, R. W. Grant, J. R. Waldrop, and S. P. Kowalczyk, Phys. Rev. Lett. 44, $1620(1980)$.

${ }^{37}$ C. Jia, Y. Chen, Y. Guo, X. Liu, S. Yang, W. Zhang, and Z. Wang, Nanoscale Res. Lett. 6, 316 (2011).

${ }^{38}$ S. J. Lee, C. Zhu, and D. L. Kwong, in Advanced Gate Stacks for HighMobility Semiconductors, edited by A. Dimoulas, E. Gusev, P. C. McIntyre, and M. Heyns (Springer, Berlin, 2007).

${ }^{39}$ S. V. Elshocht, B. Brijs, M. Caymax, T. Conard, T. Chiarella, S. D. Gendt, B. D. Jaeger, S. Kubicek, M. Meuris, B. Onsia, O. Richard, I. Teerlinck, J. V. Steenbergen, C. Zhao, and M. Heyns, Appl. Phys. Lett. 85, 3824 (2004).

${ }^{40}$ L. J. Brillson, Surfaces and Interfaces of Electronic Materials (WileyVCH, Germany, 2010).

${ }^{41}$ H. Guo, L. Liu, Z. Chen, S. Ding, H. Lu, K.-J. Jin, Y. Zhou, and B. Cheng, Europhys. Lett. 73, 110 (2006).

${ }^{42}$ M. Perego and G. Seguini, J. Appl. Phys. 110, 053711 (2011).

${ }^{43}$ M. Perego, A. Molle, and G. Seguini, Appl. Phys. Lett. 101, 211606 (2012).

${ }^{44}$ W. F. Zhang, T. Nishimula, K. Nagashio, K. Kita, and A. Toriumi, Appl. Phys. Lett. 102, 102106 (2013).

${ }^{45}$ A. K. M. Maidul Islam and M. Mukherjee, J. Phys.: Condens. Mater. 23, 435005 (2011). 